\title{
Point spread function shaping using geometric analysis
}

Bañas, Andrew Rafael; Separa, Stephen Daedalus; Engay, Einstom; Glückstad, Jesper

Published in:

Optics Communications

Link to article, DOI:

10.1016/j.optcom.2018.06.068

Publication date:

2018

Document Version

Peer reviewed version

Link back to DTU Orbit

Citation $(A P A)$ :

Bañas, A. R., Separa, S. D., Engay, E., \& Glückstad, J. (2018). Point spread function shaping using geometric analysis. Optics Communications, 427, 522-27 . https://doi.org/10.1016/j.optcom.2018.06.068

\section{General rights}

Copyright and moral rights for the publications made accessible in the public portal are retained by the authors and/or other copyright owners and it is a condition of accessing publications that users recognise and abide by the legal requirements associated with these rights.

- Users may download and print one copy of any publication from the public portal for the purpose of private study or research.

- You may not further distribute the material or use it for any profit-making activity or commercial gain

- You may freely distribute the URL identifying the publication in the public portal

If you believe that this document breaches copyright please contact us providing details, and we will remove access to the work immediately and investigate your claim. 


\section{Accepted Manuscript}

Point spread function shaping using geometric analysis

Andrew Bañas, Stephen Daedalus Separa, Einstom Engay, Jesper Glückstad

PII: $\quad$ S0030-4018(18)30558-3

DOI: $\quad$ https://doi.org/10.1016/j.optcom.2018.06.068

Reference: $\quad$ OPTICS 23255

To appear in: Optics Communications

Received date: 13 April 2018

Revised date: 19 June 2018

Accepted date : 21 June 2018

Please cite this article as: A. Bañas, S.D. Separa, E. Engay, J. Glückstad, Point spread function shaping using geometric analysis, Optics Communications (2018),

https://doi.org/10.1016/j.optcom.2018.06.068

This is a PDF file of an unedited manuscript that has been accepted for publication. As a service to our customers we are providing this early version of the manuscript. The manuscript will undergo copyediting, typesetting, and review of the resulting proof before it is published in its final form. Please note that during the production process errors may be discovered which could affect the content, and all legal disclaimers that apply to the journal pertain. 


\title{
Point spread function shaping using geometric analysis
}

\author{
Andrew Bañas ${ }^{1,2 *}$, Stephen Daedalus Separa ${ }^{2}$, Einstom Engay ${ }^{1}$ and Jesper Glückstad ${ }^{1,2}$ \\ ${ }^{1}$ Department of Photonics Engineering, Technical University of Denmark, Ørsted Plads 343, DK-2800 Kgs. Lyngby, \\ Denmark
}

${ }^{2}$ OptoRobotix ApS, DK-2000 Frederiksberg, Denmark

* Corresponding author: araf@fotonik.dtu.dk

Abstract:

\begin{abstract}
We present a method for shaping point spread functions (PSF) into simple geometric shapes that are free of noise or speckles. The derived phase distributions are intended for typical $2 f$ setups used for reading out computer generated holograms (CGH). Used in conjunction with existing CGH techniques for spot distributions the shaped PSFs can similarly be distributed on a 3D working volume. The PSF's contiguous intensity and phase make them beneficial in many applications such as photo-stimulation, optical manipulation, multi-photon excitation and laser materials processing. This manuscript presents the derivations of the phase functions and their experimental demonstration.
\end{abstract}

Keywords: Laser beam shaping; Digital holography; Fourier optics; Phase-only modulation; Spatial light modulators

\section{Introduction}

Laser beam shaping has established itself as an enabling tool in diverse applications such as optical trapping and manipulation, materials processing, atomic physics, and more recently, neurophotonics. Many of these applications require light to be shaped or distributed in a particular configuration, like when steering focal spots in optical trapping, imprinting patterns in materials processing or selectively illuminating tissues in a specimen. In many of these applications, efficient utilization of the laser source is preferred and sometimes, even necessary. Hence, techniques based on phase-only modulation are preferred, especially for laser energy efficient research applications.

Phase-only spatial light modulators (SLMs) have been instrumental for such applications. A common way of utilizing SLMs is to implement a $2 \mathrm{f}$ diffractive holography setup. In this configuration, the modulated illumination reflected off an SLM is subsequently Fourier transformed by a lens to obtain a desired pattern at the lens's focal plane. To calculate the phase distribution to be encoded on the SLM, iterative, non-analytic phase-retrieval techniques such as the Gerchberg-Saxton algorithm are often used, as analytic forms are limited to a few special cases. Such techniques had their origins in crystallography where researchers originally wished to determine crystal structure through observed X-Ray diffraction patterns [1]. In its adoption for optics, "digital holography", the known pattern is the illumination and one wishes to find a phase distribution to encode on the SLM that will result in a desired Fourier or Fresnel diffraction pattern.

Iterative phase retrieval methods generally perform well in distributing spots that have enough separation among them. However, when used to produce an extended region with a defined shape, the output would often still have the appearance of distinct spots that have just been clumped up together to fill the shape's region. These aggregated spots do not necessarily have the same phase or amplitude and due to the overlap of their individual point spread functions, severe speckle noise is often the outcome. The end-result is that within a desired output shape, some regions have peak intensities that are higher than the rest while some regions have intensities that 
are close to or equivalent to zero (e.g. where phase discontinuities are located [2]). There are many applications wherein noisy $\mathrm{CGH}$-reconstructions would be problematic. For example, in biological photo-stimulation or materials processing the use of non-linear effects makes the speckled non-uniformity of a light pattern even more pronounced.

Although, generating contiguous beam shapes is straightforward with amplitude modulation, like when using an array of micro-mirrors or switching polarization in consumer video projectors, phase-only modulation has the advantage of being much more efficient. Instead of removing light by blocking, absorbing or deflection, phaseonly light shaping techniques use interference or diffraction to re-distribute an incoming light. Hence, phase-only light shaping still finds it use in many applications, especially in contemporary research involving spatial modulation of lasers.

\subsection{Taking the best of both worlds using convolution}

In our previous work we demonstrated how one can benefit from the complementary strengths of two phaseonly light shaping techniques, in particular, Generalized Phase Contrast (GPC) [3,4] and holography [5]. By themselves, these two distinct light shaping techniques both have their advantages and their limitations [6]. However an elegant hybrid approach [5,7], can simultaneously utilize GPC to shape and produce contiguous speckle free point spread functions, while digital holography distributes copies of these PSFs into a desired sample volume. Adopting a convolution-based framework wherein beam- (or PSF-) shaping and beam distribution are treated separately therefore also allows one to separately optimize the beam's interaction with the sample and its subsequent delivery or steering within the samples' working area. This convolution principle, of course, is not limited to using GPC to shape the individual beams, and there have been previous works wherein a known analytic inverse Fourier transform corresponding to a particular beam, such as an Airy, Bessel or vortex beam, is used to illuminate a CGH encoded on a phase-only SLM [8-10]. In the case of Airy and vortex beams, the setup becomes simpler since the inverse Fourier transform amplitude is also the same as or approximated by typical SLM illumination. Hence, in these cases, one only needs to add the corresponding phase function to the spot distributing CGH function. For cases wherein the illumination amplitude also needs to be modified, such as in Holo-GPC or for multiplexed Bessel beams (typically requiring a ring illumination), additional components are needed to pre-shape the beam before it illuminates the SLM.

Although such configurations can be considered as a subset of a "Bartelt tandem system" [11,12], these approaches take advantage of convolution to illuminate the SLM with a simpler well defined beam instead of a more complex inverse Fourier transform of an arbitrary target output shape. Besides having a simpler form, the use of the same illumination across different output distributions allows one to use fixed static optical elements instead of an additional SLM (or a designated region within the same SLM [13]) as in typical direct realizations of a so-called Bartelt-configuration. For example, in Holo-GPC [5], we can use a static phase mask to efficiently generate the Airy-like illumination for the SLM. Similarly, fixed axicon lenses have been used to holographically multiplex Bessel beams [8].

Unfortunately, having a different SLM illumination besides the typical Gaussian or tophat imposes more constraints on the optical setup and can limit the potential applications. For example, it is required that the inverse Fourier transformed profile fits within the SLM aperture to prevent the loss of higher spatial frequencies from the output. On the other hand, making this illumination profile narrower increases its peak intensity, which in turn, might potentially damage the SLM. Aperture constraints, additional components and peak intensity constraints therefore make it preferable if typical laser source profiles can be used directly for SLM readout as also already commonly practiced in a majority of CGH applications. At the same time, it is also desirable if the typical noise from the CGH-output can be reduced or eliminated.

\section{Mapping beams into simple and practical geometrical forms}


In addition to the well-known diffractively generated beams, simple shapes characterized by an extended area wherein the intensity is ideally uniform easily lend themselves to a wide variety of practical applications. For example, simple disks or square patterns have straightforward applications in biophotonics, like for selective tissue stimulation $[14,15]$ or counter-propagating optical traps [16-18] or in materials processing to get more uniform ablation profiles [19-21].

The following sections thus outline how we derive a phase function that allows an input Gaussian beam to be transformed into simple geometric shapes such as disks and rectangles.

\subsection{Approximate geometric mapping for shaping Point Spread Functions}

We derive our SLM phase functions using a refractive or geometric mapping conceptual framework similar to [22-25]. However, instead of using the more rigorous mathematical derivations presented in prior work, we utilize the grating approach commonly used in diffractive beam steering as the basis of our derivations. In diffractive optics, a planar, prism or "mirror" phase with a defined slope at the input plane is used to map a point at the Fourier plane. This so-called "lenses and prisms" approach for actuating diffractive spots removes the need of time-consuming iterative methods, hence allowing real time optical trapping.

We extend this SLM-to-spot mapping principle by considering the whole SLM area as a collection of tilt-able mirrors each having an independent slope. Similarly, the output shape is considered as a collection of adjacent focal spots. These assumptions are illustrated in Figure 1. As illustrated, unlike in iterative methods, a local region within the SLM has a direct mapping to a local subdivision of the output extended shape.

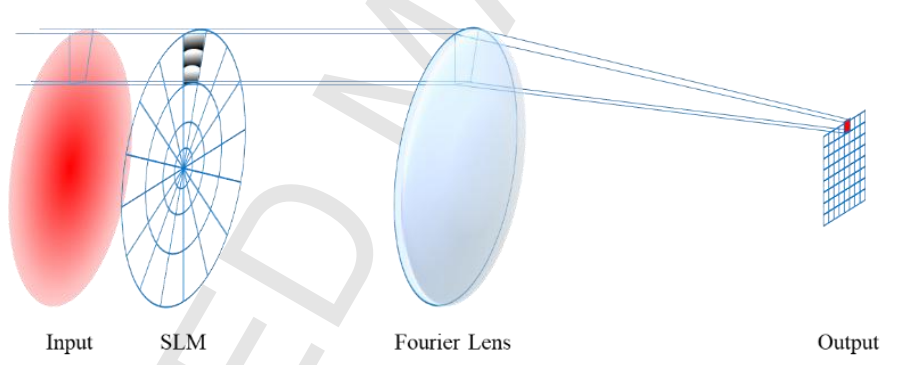

Fig. 1. Geometric mapping principle. A SLM subdivides input Gaussian laser illumination into sub-regions that uniquely map into sub-regions that form the output shape. The phase profile in a given input sub-region at the SLM is determined by the location of the corresponding output sub-region while the relative area is determined by the desired intensity level at that output region. (Sub-divisions are arbitrarily simplified and not drawn to scale).

Imposing that the energy is conserved from the SLM region and to the point in the PSF where it is mapped to, allows the derivation of a mapping between focal plane coordinates and SLM-coordinates. Thus, the problem can be formulated as finding the mapping function

$$
r_{f}\left(R_{S L M}\right)
$$

where, $r_{f}$ denotes positons at the focal plane and $R_{S L M}$ denotes positions at the SLM. Once this function is solved a "localized" planar phase at the region $R_{S L M}$ can be made to focus at $r_{f}$, similarly to how a prism phase actuates a single spot:

$$
\varphi\left(R_{S L M}\right)=\frac{2 \pi}{\lambda f} R_{S L M} r_{f}\left(R_{S L M}\right)
$$

In the above expression, $\lambda$ denotes the SLM's illumination wavelength and $f$ is the focal length of the lens that performs the optical Fourier transform of the complex light field at the SLM.

\subsection{Circular output}


We start by assuming that the SLM is illuminated with a Gaussian beam. For simplicity, we assume that the output circle has uniform intensity within its area (though the actual result deviates from this assumption). Given a target output circle with radius $a$, and a Gaussian illumination whose half beam waist is $w_{0}$, an expression for the energy conservation relation is given by:

$$
\pi w_{0}^{2}=\pi a^{2}
$$

If we scale the total energy to a fractional region bounded by the corresponding SLM and focal plane radiuses, we obtain the following expression:

$$
\frac{\pi w_{0}^{2}\left(1-e^{-\frac{R^{2}}{w_{0}^{2}}}\right)}{\pi w_{0}^{2}}=\frac{\pi r^{2}}{\pi a^{2}}
$$

Solving for $R_{S L M}$ in terms of $r_{f}$ and using Eq. (2) for the expression for the phase, we get:

$$
\varphi(R)=\frac{2 \pi R}{\lambda f} a \sqrt{\left(1-e^{-R^{2} / w_{0}^{2}}\right)}
$$

Figure 2 shows a numerically simulated result performed with a $1024 \times 1024$ discrete Fourier transform. The Gaussian waist used is 300 samples, and a disk radius of 50 samples is used to calculate the phase.
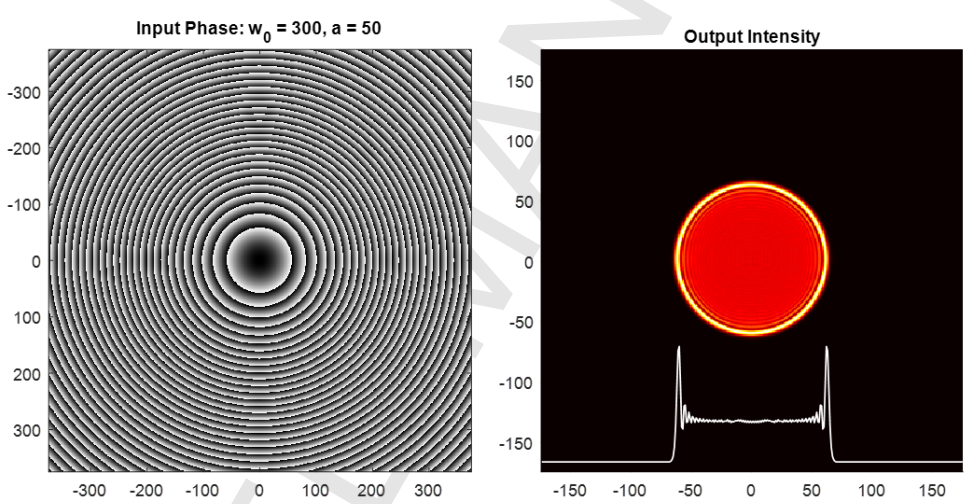

Fig. 2. Simulated input phase and output intensity distributions for generating a disk-shaped PSF.

\subsection{Rectangular output}

A Gaussian beam profile bounded by a rectangle can also be treated analytically using error functions. When integrating a Gaussian over rectangular bounds $A \times B$ (e.g. the SLM boundaries) and comparing with a rectangular area $a \times b$ with uniform intensity, the following expression can be derived:

$$
\left[\frac{\sqrt{\pi}}{2} w_{0} \operatorname{erf}\left(\frac{A}{w_{0}}\right)\right] \times\left[\frac{\sqrt{\pi}}{2} w_{0} \operatorname{erf}\left(\frac{B}{w_{0}}\right)\right]=a \times b
$$

Since the terms are separable in $x$ and $y$, a simpler relation along one dimension can be used instead,

$$
\frac{\sqrt{\pi}}{2} w_{0} \operatorname{erf}\left(\frac{A}{w_{0}}\right)=a
$$

Like with the circle, we assume correspondence in the energy covered by a sub-rectangle at the input with length $X$ and sub-rectangle at the output with length $x_{f}$,

$$
\frac{\frac{\sqrt{\pi}}{2} w_{0} \operatorname{erf}\left(\frac{X}{w_{0}}\right)}{\frac{\sqrt{\pi}}{2} w_{0} \operatorname{erf}\left(\frac{A}{w_{0}}\right)}=\frac{x_{f}}{a}
$$


From the above expression we can solve for the focal point location $x_{f}$ where the SLM location $X$ should be mapping to:

$$
x_{f}(X)=\frac{\operatorname{erf}\left(\frac{X}{w_{0}}\right)}{\operatorname{erf}\left(\frac{A}{w_{0}}\right)} a
$$

Hence, the phase along $x$ function is given by

$$
\varphi_{x}(X)=\frac{2 \pi}{\lambda f} X \frac{\operatorname{erf}\left(\frac{X}{w_{0}}\right)}{\operatorname{erf}\left(\frac{A}{w_{0}}\right)} a
$$

A similar expression is obtained for the other direction by respectively replacing $x, X, A, a$ with $y, Y, B, b$. The final phase encoded on the SLM would thus be

$$
\varphi(X, Y)=\varphi_{x}(X)+\varphi_{y}(Y)
$$

Figure 3 shows a numerically simulated result performed with a $1024 \times 1024$ discrete Fourier transform. The Gaussian waist used is 300 samples, and the square dimensions used to calculate the phase are $35 \times 35$ samples.
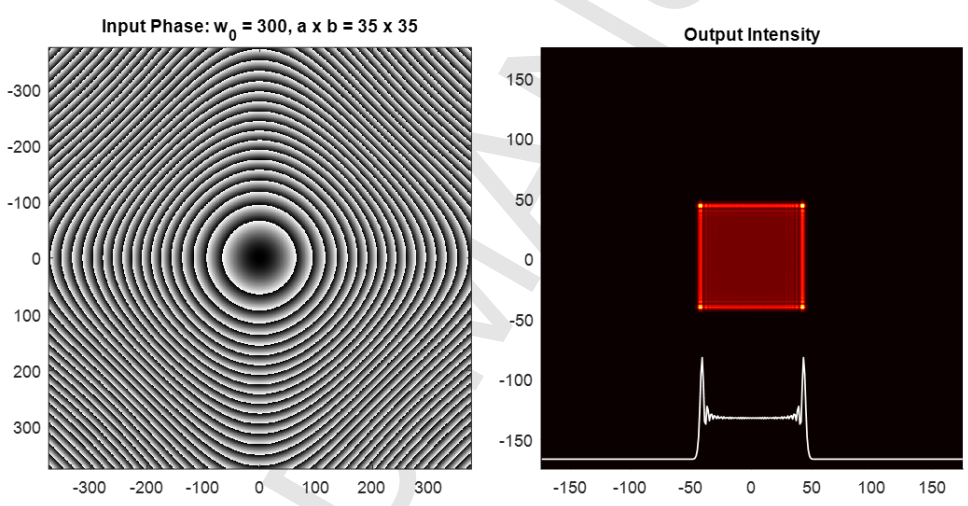

Fig. 3. Simulated input phase and output intensity distributions for generating a square PSF.

Due to the alternate basis of our derivation, we arrive at mathematically different phase functions from those presented in existing literature $[22,23,25]$, despite general visual similarities of the encoded phase functions (i.e. in Fig. 2 and Fig. 3). Furthermore, our approach takes advantage of the dynamic nature of SLMs by making it straightforward to change the dimensions of the output shape.

\subsection{Limits of the geometric approximation}

The presented approach only uses geometrical assumptions and ignores diffraction effects. Nonetheless, in simple cases, e.g. for disks and rectangles, as presented here, the fidelity of the output shapes is already good enough for many SLM applications. In addition to its calculation speed the sculpted shapes are also far better than what can be obtained through common iterative and computationally demanding approaches. The approach also bears similarity to refractive beam shaping [24], but relies on an SLM surface and a Fourier transforming lens instead of fabricated freeform refractive optical elements.

One immediately observed effect is the ringing at the edges of the generated output shapes. This results from the fact that the actual points assumed to build up the overall shaped output also have their own point spread functions. Adjacently mapped focal points can thus overlap and interfere, resulting in an intensity that is lower than what is initially assumed in the derivations. Moreover, as there are less overlapping mapped points towards the shape boundary to interfere with each other, the light intensity at the edges become higher. This "constituent 
PSF" overlapping is also true for iteratively generated CGH-outputs. However, in the case of geometric mapping, the phases of adjacent points vary in a continuous way that prevents abrupt destructive interference within the output shape's area.

One way to minimize the intensity at the shape edges is to consider them as high frequency artifacts. A low-pass effect may be achieved if the illuminating Gaussian beam is smaller than the one designed for. However, instead of decreasing the size of the Gaussian beam, the phase pattern on the SLM can simply be enlarged such that it is supposedly calibrated for the Gaussian beam that is larger than that available in the experiment. As the edges of the SLM are mapped into the edges of the output shape, this workaround can also be thought of as minimizing edge contributions to suppress the ringing. Besides effective beam size adjustments used in this work, alternative solutions might modify the phase function towards the edge of the SLM. In experiments, the limited aperture sizes also contribute in suppressing the edge ringing.

\subsection{Comparison to other beam shaping approaches}

Other similar analytic approaches used for transforming a typically Gaussian beam into simple geometries include Eikonal or stationary phase based approaches [23,25,26], refractive mapping [24], and ray tracing [27]. Unlike iteratively generated holograms, these analytic/semi-analytic approaches are characterized by typically slowly varying phase distributions which redirects portions of the input beam in a very well-defined manner. The rectangle-generating phase function we have presented has similar terms to what has been derived in [23] using the Eikonal method and might be interpreted as a simplified version that is practically geared towards $2 f$-focusing systems found in computer generated holography. Furthermore, compared to [23], the output shape from our derived phase functions form sharper boundaries close to the intended focal plane.

We have also recently proposed how to holographically distribute multiple copies of GPC-generated speckle-free shapes with a hybrid of holography and GPC coined "Holo-GPC". For certain tradeoffs, our PSF shaping approach overcomes several notable limitations of Holo-GPC. Holo-GPC's use of a diffraction output to illuminate an SLM means that there is a relatively strong zero order within the illumination that is potentially more likely to reach the damage threshold of the SLM. The PSF shaping uses the Gaussian illumination directly hence no SLM pixels are receiving significantly higher power than the rest. This also means that no additional component is placed before the SLM, whereas Holo-GPC would typically require a static phase mask and a lens to be added to the standard holography setup. Perhaps the biggest advantage over Holo-GPC is that, for the same setup, the PSFshaping approach provides freedom over the PSF size and shape as it is not depending on a static fabricated phase mask (assuming only one SLM is used). On the other hand, the shapes that can actually be realized with the PSF-shaping approach is much more limited in comparison to the near arbitrary phase masks that GPC can utilize. In Holo-GPC it is possible to revert to strongly focused spots by adopting matched filtering GPC (mGPC) $[28,29]$ to add concentric rings on the SLM. In this approach, one only has to "turn off" the PSF phase function to revert to the usual holographic focusing.

In Holo-GPC changing the PSF-shapes require changing the input phase masks to change the illumination distribution at the SLM. This new PSF-shaping approach can take advantage of the fact that the same Gaussian beam input is used for different PSF-shapes. Hence, within limited quantities, one could multiplex different PSFshapes by randomly subdividing the SLM-region [30] to correspond to different PSF-shapes reconstructed at different locations. In short, different PSF-shapes can be reconstructed simultaneously. Combining different basic shapes can be used to build relatively more complex patterns as will be shown in the experiments. PSF-shaping, however, is limited by simple PSF-shapes whereas Holo-GPC can utilize arbitrarily shaped phase masks.

\section{Experimental results}


The optical setup is based on a standard $2 f$ holographic setup that can also be used to project Fourier holograms calculated with existing methods. The used LCoS-SLM has an active area of $16 \times 12 \mathrm{~mm}$ containing $800 \times 60020 \mu \mathrm{m}-$ pixels and is illuminated by a Gaussian beam having a $635 \mathrm{~nm}$ wavelength and $\sim 6 \mathrm{~mm}$ diameter. To better visualize the output shapes, we have used long focal lengths that result in a wider Fourier distribution where the capturing device is directly placed. For more close-up views we have used a 350mm focal length lens and for wider views, we used $200 \mathrm{~mm}$. Based on observations, the dimensions of the target shapes should be at least $\sim 10$ times that of the produced diffraction limited spot, otherwise, the shapes become less defined. Similar size constraints have also been mentioned in [23]. We have experimented with phase functions for generating a circle, a square and a rectangle with a large (1:10) aspect ratio. For comparison, results produced using the iterative Gerchberg-Saxton algorithm are also produced on the same setup. Results in Figs. 4(a)-(b) and 4(d)-(e) were formed with the 350mm focal length lens for a more close up view, while the large aspect ratio output in Figs. 4(c) and 4(f) were formed with the $200 \mathrm{~mm}$ focal length lens. Besides taking longer to calculate, the GS results show the usual noise and discontinuities.
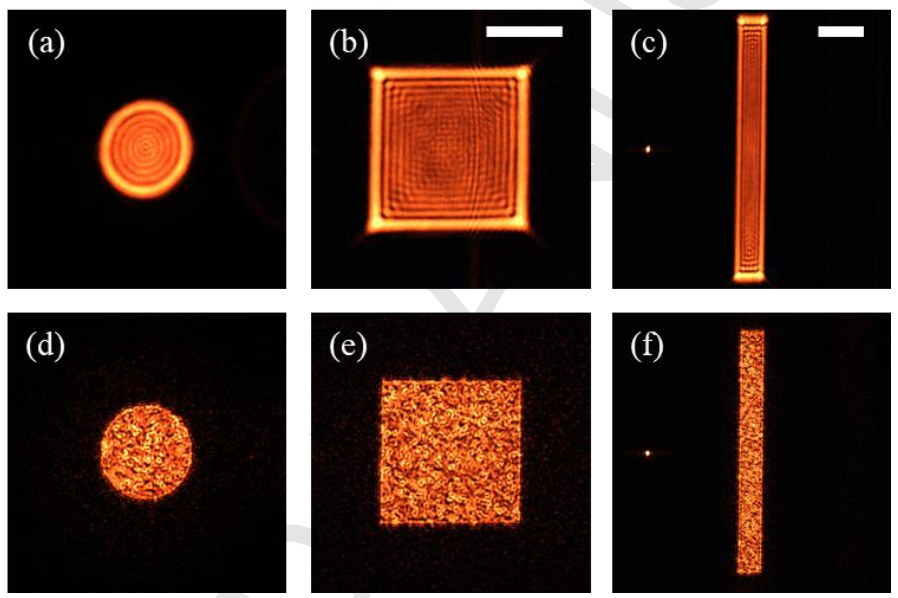

Fig. 4. Extended geometric shapes generated in the same 2f-setup using SLM phase distributions generated by the PSF-shaping method (a-c) and the Gerchberg-Saxton algorithm (d-f). For reference, the zero order spot is left visible in (c) and (f). Scalebar is $1 \mathrm{~mm}$ in the camera.

The practical utility of the shaped PSFs is realized when convolving with a hologram for distributing spots. For example, the same GS algorithm can be used to generate a spot array as shown in Figure 5 . To implement convolution, the analytically derived PSF shaping phase is simply added to the numerically obtained CGH. Since the spot array $\mathrm{CGH}$ calculation did not take the modulation transfer function into account, there is an observable graduation of intensity with the shaped beams further from the zero order. The same convolution principle also applies for CGHs that produce 3D spot distributions. In Figure 6(a)-(c), one can see how output squares are imaged at 3 separate planes spaced $2.7 \mathrm{~cm}$ apart).
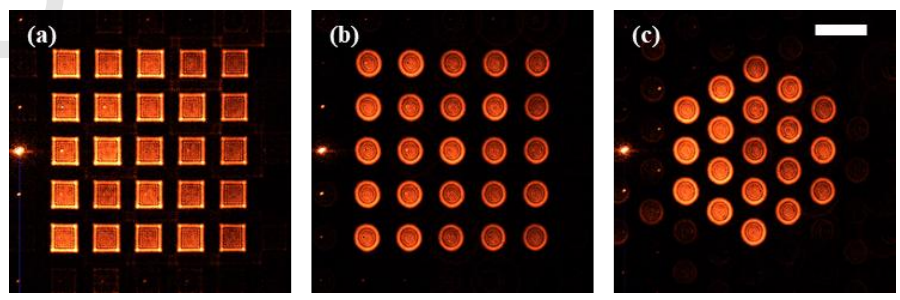

Fig. 5. PSFs multiplexed using an array generating CGH. Scalebar is $1 \mathrm{~mm}$ in the camera.

Real time algorithms for distributing spots [30] can be used to re-configure the shaped PSFs for applications such as optical manipulation or cell sorting. As this spot actuation algorithm works by spatially dividing the SLM to 
independent regions assigned to independent spots, this can also be used to encode independent PSFs. Each spatially displaced PSF can thus have a different shape or size while sharing the same illumination and SLM. Figure 6 (d)-(f) demonstrates how different PSF-shapes can be multiplexed. Unfortunately, the nature of this multiplexing scheme introduces noise that becomes more prominent as more independent foci are encoded on the SLM. Furthermore, unlike with spots intended for this multiplexing scheme, the relatively lower peak intensities within the PSF's extended region are closer to the noise floor.
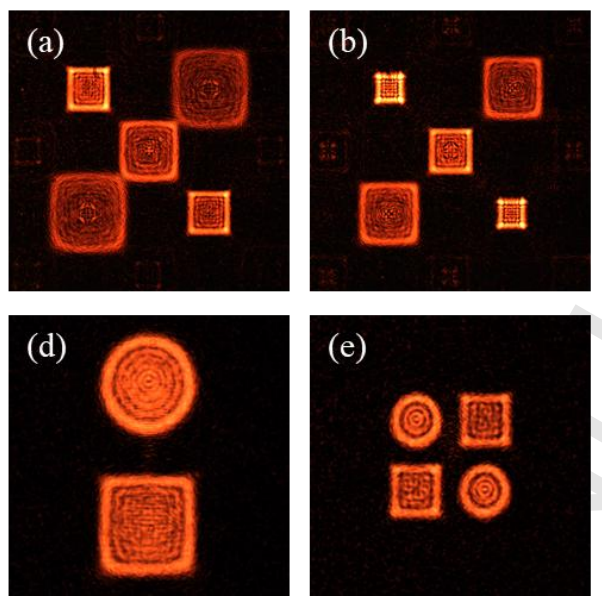

Fig. 6. Addressing a square PSF into different axial planes (a-c) spaced $2.7 \mathrm{~cm}$ apart. Different PSF shapes are multiplexed by subdividing the SLM into non overlapping areas assigned to each corresponding PSF phase function (d-f). Scalebar is $1 \mathrm{~mm}$ in the camera.

\section{Conclusions}

This work presents an approximate analytic approach for shaping Point Spread Functions (PSFs) that are free of noise or speckles. The derived phase functions for these PSFs are relatively easy to calculate and implement. Contiguous extended optical regions are beneficial in applications such as photo-stimulation, optical manipulation, laser materials processing amongst others. The presented method makes it practical to use an SLM setup that is already typically used for diffractive computer generated holography. As with our previous work [5], distinguishing the roles of beam shaping and beam distribution enables one to treat PSF-engineering as a separate task while taking advantage of existing diffractive holography techniques to multiplex the shaped PSFs. Future work might include the possible use of numerical techniques to improve the output uniformity or investigate the extension of the geometric mapping principle to the third (axial) dimension to allow more versatile light distributions.

\section{Acknowledgements}

We acknowledge the support of the Lundbeck Foundation, the Combined Molecular Microscopy for Therapy and Personalized Medication in Rare Anaemias Treatments (CoMMiTMent) FP7 collaborative project (Grant agreement number: 602121), and the Regulation of red cell life-span, erythropoiesis, survival, senescence and clearance (Project ID 675115) Horizon-2020 training network.

\section{References}

1. R. W. Gerchberg and W. O. Saxton, "A practical algorithm for the determination of the phase from image and diffraction plane pictures," Optik (Stuttg). 35, 237-246 (1972).

2. M. Guillon, B. C. Forget, A. J. Foust, V. De Sars, M. Ritsch-Marte, and V. Emiliani, "Vortex-free phase profiles for uniform patterning with computer-generated holography," Opt. Express 25, 12640-12652 
(2017).

3. J. Glückstad and D. Z. Palima, Generalized Phase Contrast: Applications in Optics and Photonics (Springer Series in Optical Sciences, 2009).

4. A. Bañas, D. Palima, M. Villangca, T. Aabo, and J. Glückstad, "GPC light shaper for speckle-free one-and two-photon contiguous pattern excitation," Opt. Express 7102, 5299-5310 (2014).

5. A. Bañas and J. Glückstad, "Holo-GPC: Holographic Generalized Phase Contrast," Opt. Commun. 392, 190195 (2017).

6. D. Palima and J. Glückstad, "Comparison of generalized phase contrast and computer generated holography for laser image projection," Opt. Express 16, 5338-49 (2008).

7. A. Bañas and J. Glückstad, "Light Shaping with Holography, GPC and Holo-GPC," Opt. Data Process. Storage 3, 20 (2017).

8. T. Cizmár, V. Kollárová, X. Tsampoula, F. Gunn-Moore, W. Sibbett, Z. Bouchal, and K. Dholakia, "Generation of multiple Bessel beams for a biophotonics workstation.," Opt. Express 16, 14024-35 (2008).

9. X.-Z. Wang, Q. Li, Z.-P. Xiong, Z. Zhang, and Q. Wang, "Generation and scanning of Airy beams array by combining multiphase patterns," Appl. Opt. 52, 3039-3047 (2013).

10. X. Yu, R. Li, S. Yan, B. Yao, P. Gao, G. Han, and M. Lei, "Experimental demonstration of 3D accelerating beam arrays," Appl. Opt. 55, 3090-3095 (2016).

11. H. O. Bartelt, "Computer-generated holographic component with optimum light efficiency.," Appl. Opt. 23, 1499 (1984).

12. H. O. Bartelt, "Applications of the tandem component: an element with optimum light efficiency.," Appl. Opt. 24, 3811 (1985).

13. A. Jesacher, C. Maurer, A. Schwaighofer, S. Bernet, and M. Ritsch-Marte, "Near-perfect hologram reconstruction with a spatial light modulator.," Opt. Express 16, 2597-603 (2008).

14. E. Papagiakoumou, "Optical developments for optogenetics.," Biol. Cell 105, 443-64 (2013).

15. E. Papagiakoumou, F. Anselmi, A. Bègue, V. de Sars, J. Glückstad, E. Y. Isacoff, and V. Emiliani, "Scanless two-photon excitation of channelrhodopsin-2.," Nat. Methods 7, 848-54 (2010).

16. T. Aabo, A. R. Banás, J. Glückstad, H. Siegumfeldt, and N. Arneborg, "BioPhotonics workstation: a versatile setup for simultaneous optical manipulation, heat stress, and intracellular $\mathrm{pH}$ measurements of a live yeast cell.," Rev. Sci. Instrum. 82, 083707 (2011).

17. H.-U. Ulriksen, J. Thogersen, S. Keiding, I. R. Perch-Nielsen, J. S. Dam, D. Z. Palima, H. Stapelfeldt, and J. Glückstad, "Independent trapping, manipulation and characterization by an all-optical biophotonics workstation," J. Eur. Opt. Soc. Rapid Publ. 3, 08034 (2008).

18. S. Tauro, A. Bañas, D. Palima, and J. Glückstad, "Dynamic axial stabilization of counter-propagating beamtraps with feedback control.," Opt. Express 18, 18217-22 (2010).

19. D. Liu, Y. Wang, Z. Zhai, Z. Fang, Q. Tao, W. Perrie, S. P. Edwarson, and G. Dearden, "Dynamic laser beam shaping for material processing using hybrid holograms," Opt. Laser Technol. 102, 68-73 (2018).

20. Z. Kuang, J. Li, S. Edwardson, W. Perrie, D. Liu, and G. Dearden, "Ultrafast laser beam shaping for material processing at imaging plane by geometric masks using a spatial light modulator," Opt. Lasers Eng. 70, 1-5 (2015).

21. S. Rung, J. Barth, and R. Hellmann, "Characterization of Laser Beam Shaping Optics Based on Their Ablation Geometry of Thin Films," Micromachines 5, 943-953 (2014).

22. F. M. Dickey, Laser Beam Shaping: Theory and Techniques (CRC press, 2014).

23. M. Duparré, M. A. Golub, B. Lüdge, V. S. Pavelyev, V. A. Soifer, G. V Uspleniev, and S. G. Volotovskii, "Investigation of computer-generated diffractive beam shapers for flattening of single-modal $\mathrm{CO} 2$ laser beams," Appl. Opt. 34, 2489-2497 (1995).

24. J. A. Hoffnagle and C. M. Jefferson, "Design and performance of a refractive optical system that converts a Gaussian to a flattop beam.," Appl. Opt. 39, 5488-99 (2000).

25. L. A. Romero and F. M. Dickey, "Lossless laser beam shaping," JOSA A 13, 751-760 (1996).

26. M. A. Golub and I. Grossinger, "Diffractive optical elements for biomedical applications," in Biomedical 
Systems and Technologies II (1998), Vol. 3199, pp. 220-232.

27. E. Sidick, S. D. Collins, and a Knoesen, "Trapping forces in a multiple-beam fiber-optic trap.," Appl. Opt. 36, 6423-33 (1997).

28. J. Glückstad and D. Palima, "Combining Generalized Phase Contrast with matched filtering into a versatile beam shaping approach," J. Phys. Conf. Ser. 206, 012006 (2010).

29. A. Bañas, D. Palima, and J. Glückstad, "Matched-filtering generalized phase contrast using LCoS picoprojectors for beam-forming." Opt. Express 20, 9705-12 (2012).

30. M. Montes-Usategui, E. Pleguezuelos, J. Andilla, and E. Martín-Badosa, "Fast generation of holographic optical tweezers by random mask encoding of Fourier components," Opt. Express 14, 2101-2107 (2006). 